\title{
Anabases
}

ANABASES Traditions et réceptions de l'Antiquité

$4 \mid 2006$

Varia

\section{Synthèse et bilan d'une exploration préliminaire}

\section{Geneviève Gal}

\section{(2) OpenEdition}

Journals

Édition électronique

URL : http://journals.openedition.org/anabases/3008

DOI : 10.4000/anabases.3008

ISSN : 2256-9421

\section{Éditeur}

E.R.A.S.M.E.

\section{Édition imprimée}

Date de publication : 1 octobre 2006

Pagination : 257-258

ISSN : 1774-4296

\section{Référence électronique}

Geneviève Gal, « Synthèse et bilan d'une exploration préliminaire », Anabases [En ligne], 4 | 2006, mis en ligne le 01 janvier 2012, consulté le 21 octobre 2019. URL : http://journals.openedition.org/ anabases/3008; DOI : 10.4000/anabases.3008 
Anabases 4 (2006), p. 257-258

\section{Synthèse et bilan d'une exploration préliminaire}

GENEVIÈVE GAL

$\mathrm{C}$ ETTE JOURNÉE fut particulièrement enrichissante car nombre de participants (intervenants, invités, simples auditeurs) ont alimenté les discussions. Cette dynamique, finalement peu courante dans ce genre de manifestation, s'explique par le fait que les sujets abordés portaient moins sur des points de connaissance très pointue que sur l'évaluation de la pertinence même du projet et les possibles modalités de sa mise en œuvre.

La principale question soulevée fut celle de savoir si en définitive les différentes interventions ne montraient pas que la seule entreprise, aujourd'hui véritablement acceptable scientifiquement, était de s'en tenir à une étude historiographique de l'ouvrage et d'entreprendre parallèlement une recherche sur son iconographie (recherche des références et des localisations d'objets, évaluation de la rigueur des reproductions fournies, etc.). Les tenants de cette option minimale estimaient principalement que la réalisation d'une encyclopédie savante correspond aujourd'hui à un concept scientifiquement dépassé, d'autant plus que ce genre d'outil existe déjà (la Realencyclopädie en particulier fut évoquée).

L'expression de ces réticences nous a permis d'avancer dans nos réflexions et de présenter plus clairement les objectifs de notre projet. Tout d'abord, il convient de rappeler qu'il n'a jamais été question de réécrire les articles du vieux Daremberg et Saglio, ce qui pour diverses raisons est impensable aujourd'hui, mais de les commenter sur les points qui sont de toute évidence dépassés; c'est pour cette raison que nous avions placé dans la version actuelle du site un onglet ouvert à tous "Commentaires libres »; il devait servir d'espace expérimental dans la perspective d'une future révision produite par les savants participant à la deuxième phase du projet.

Par ailleurs, s'il est vrai qu'il existe déjà d'excellentes encyclopédies de l'Antiquité, aucune n'est identique à ce que nous voulons construire, qui permette de mettre à la disposition du public des commentaires évolutifs et une documentation toujours plus 
riche en rapport avec les articles du Dictionnaire des Antiquités. Il s'agit non seulement de prendre en compte les apports d'un siècle de travaux sur l'Antiquité et de découvertes archéologiques, mais aussi de rester ouvert à toutes les nouveautés car le support requis autorise en permanence les ajouts. Actuellement, il n'existe que des ouvrages imprimés, avec toutes les limitations de consultation et d'actualisation que cela suppose, tandis que notre projet repose sur les capacités immenses de stockage et sur la souplesse d'édition et de diffusion de l'Internet (gratuité, rapidité, accessibilité permanente sans déplacement et potentiel évolutif). Outre son intérêt pour les spécialistes, il doit permettre d'assurer la promotion des Sciences de l'Antiquité en favorisant le développement de l'environnement socioculturel propice, socle indispensable de la recherche, dans un contexte de désaffection des études classiques traditionnelles.

Concrètement, dans les semaines qui ont suivi cette fructueuse journée, afin de répondre à un appel à projet lancé par l'ANR (Agence Nationale pour la Recherche), nous avons précisé nos objectifs et mis en place une organisation permettant de progresser, ceci en tenant compte des pistes évoquées lors des débats ; désormais notre entreprise porte un nom plus en accord avec son contenu : le ND@GR en ligne (Nouveau $D @_{G R}$ en ligne).

Quatre équipes de recherche (CRATA, ERASME, UTAH, relayées par la MSH de Toulouse, et CEPAM rattaché à la MSH de Nice) vont unir leurs efforts pour construire ce nouvel outil dont la qualité scientifique sera garantie par un Conseil scientifique international constitué de savants reconnus dans différentes spécialités des études antiques ${ }^{1}$. C'est avec la plus grande impatience que nous attendons la réponse de l'ANR afin de fournir, dans ces colonnes, des détails plus amples sur la manière dont nous avons conçu la suite de notre entreprise.

\begin{tabular}{l} 
GENEVIÈVE GAL \\
\hline Université de Toulouse II - Le Mirail \\
UFR d'Histoire, Arts et Archéologie \\
5, allées Antonio Machado \\
F-31058 Toulouse \\
c_gal@club-internet.fr
\end{tabular}

1 Présidente d'honneur : Aline Rousselle ; membres: Jean Andreau, Pascal Arnaud, Germaine Aujac, Giancarlo Mazzoli, Hinnerk Bruhns, Olivier Devillers, Claude Domergue, Monique Dondin-Payre, Jonathan Edmondson, Abdelmajid Ennabli, MarieHélène Garelli, Andrea Giardina, Carlos Gomez-Bellard, Anne-Françoise Jaccottet, Christian Jacob, Bernard Legras, François Lissarrague, Léopold Migeotte, Pascal Payen, Nicholas Purcell, Bruno Rochette, Stéphane Verger. 